\title{
Vergessene Heilpflanzen
}

Klaus Peter Latté

\section{ZUSAMMENFASSUNG}

Pflanzen wurden zu allen Zeiten der Menschheit als Lebensmittel und als Arzneimittel angewendet. In der Antike und später im Mittelalter wurden zahlreiche Pflanzen und deren arzneiliche Verwendung beschrieben. Aus verschiedenen Gründen, u.a. das Aufkommen von synthetischen Wirkstoffen ab dem 19. Jahrhundert, ist das Wissen um pflanzliche Arzneimittel zum Teil verloren gegangen. In diesem Beitrag wird der Weg vom Altertum bis zur heutigen Zeit anhand von fünf Arzneipflanzen bzw. -drogen, Blutweiderichkraut, Tormentillwurzelstock, Alantwurzel, Leberblümchenkraut und den Knollen des Blauen Eisenhut verfolgt und deren derzeitige Anwendung betrachtet.

\section{Schlüsselwörter}

Lythrum salicaria, Potentilla erecta, Inula helenium, Hepatica nobilis, Aconitum napellus, vergessene Heilpflanzen, traditional use, well-established use, rationale Phytotherapie
Pflanzen waren zu allen Zeiten der Menschheitsgeschichte als Lebensmittel und als Heilmittel in der Volksheilkunde bzw. Medizin von großer Bedeutung. Seit der Antike sind hunderte von Heilpflanzen bekannt, die jedoch zum größten Teil jetzt nicht mehr angewendet werden und scheinbar aus dem Blickfeld unserer Zeit verschwunden sind. Einige dieser altbekannten Heilpflanzen bzw. Arzneidrogen, wie beispielsweise die Weidenrinde, Johanniskraut oder Leinsamen, werden heute auch im Rahmen der rationalen Phytotherapie angewendet, da die Wirksamkeit dieser Pflanzen in klinischen Studien belegt ist und zugleich die Qualität und Unbedenklichkeit gesichert ist, weitere als traditionelle pflanzliche Arzneimittel. In diesem Beitrag soll der Frage nachgegangen werden, was mit vielen anderen, in der Antike und im Mittelalter beschriebenen Pflanzen geschehen ist und warum diese heute nicht mehr oder nur noch in geringem Maße arzneiliche Verwendung finden. Anhand von fünf Heilpflanzen soll der Weg in die heutige Zeit exemplarisch verfolgt und der Kenntnisstand zur Anwendung dieser Pflanzen und deren Drogen zusammengefasst werden.

\section{Geschichtliches im Überblick}

Ursprünglich wurde das Wissen um die Anwendung von Heilpflanzen mündlich von Generation zu Generation weitergegeben. Die frühesten schriftlichen Überlieferungen sind etwa 5000 Jahre alt und stammen von den Sumerern [4]. Im europäischen Raum gibt es medizinische Überlieferungen aus der Antike, u. a. von Hippokrates (459-370 v. Chr.) und von Plinius d.Ä. (23-79 n. Chr.), in denen zahlreiche Arzneipflanzen und deren Anwendung beschrieben werden. Eine sehr umfassende Zusammenstellung von ca.
1000 Arzneidrogen, darunter mehr als 600 pflanzlichen Drogen, erstellte der griechische Arzt Dioskurides im 1. Jahrhundert n.Chr., die heute in Form von Abschriften und Überarbeitungen noch erhalten ist (u.a. bekannt als „Wiener Dioskurides“). Im Mittelalter, bis etwa zum 12. Jahrhundert, wurde das Wissen um die Anwendung von Heilpflanzen durch die Klostermedizin, u.a. durch die Schriften der Äbtissin Hildegard von Bingen (1098-1179), und danach durch verschiedene Kräuterbücher bis zum Jahr 1500 weitergegeben. Durch die Erfindung der Buchdruckerkunst durch Johannes Gutenberg Mitte des 15. Jahrhunderts war eine größere Verbreitung der Kräuterbücher möglich. Eine wesentliche Erweiterung ist den „Vätern der Botanik“ Otto Brunfels (1488-1534), Hieronymus Bock (1498-1554) und Leonhart Fuchs (1501-1566) zu verdanken, da die Angaben über die arzneiliche Verwendung der pflanzlichen Drogen durch Abbildungen der Pflanzen in Form von Holzschnitten ergänzt waren. So erschienen kurz hintereinander zwischen den 1530erund 1540er-Jahren die Kräuterbücher von Brunfels, Bock und Fuchs mit jeweils einigen hundert Abbildungen [10]. In einem Kräuterbuch von Tabenaemontanus (15251590) aus dem Jahr 1588 waren bereits ca. 3000 Pflanzenarten abgebildet und deren arzneiliche Anwendung beschrieben [5]. Bedingt durch Handelsreisen, durch Expeditionen und später auch durch die Kolonialisierung wurden pflanzliche Drogen aus anderen Kontinenten in Europa eingeführt, u.a. Echinacea, Chinarinde, Ipecacuanhawurzel und Ratanhiawurzel [4]. Erst im 19. und 20. Jahrhundert wurde aus pflanzlichen Drogen Inhaltsstoffe isoliert, hinzu kamen neue synthetische Wirkstoffe, sodass die Anwendung von Heilpflanzen nach und nach an Bedeutung verlor [10]. 


\section{Der Gewöhnliche Blutweiderich - Lythrum salicaria L.}

Lythrum salicaria L. stammt aus der Familie der Lythraceae (Weiderichgewächse) und kommt auch in Europa vor, insbesondere in Feuchtgebieten wie Flussufern, Teichen und Flachmooren. Die Staude mit einem dicken Wurzelstock wird bis zu 1,5 m hoch und hat lanzettliche Laubblätter und einen ährenförmigen Blütenstand, wobei die rot-violetten Kronblätter in der Blütezeit von Juni bis September auffallen (\Abb.1). Arzneilich werden seit langem die getrockneten, blühenden Zweigspitzen, Lythri herba, verwendet. Bereits in der Antike war diese Pflanze zur Behandlung von Ekzemen bekannt, aber auch zur Behandlung von Blutspeien, Ruhr und Metrorrhagie [30]. In den Kräuterbüchern des 16. Jahrhunderts wird vom Einsatz dieser Droge bei Hämorrhagien im Verdauungstrakt und in der Nase, von der Wundbehandlung, von Menorrhagie (Verlängerung der Monatsblutung) und von Dysenterien berichtet. Im 18. und 19. Jahrhundert wurden Zubereitungen aus L. salicaria zudem äußerlich bei Augenentzündungen, Sinusitis, Krampfadern, Hämorrhoiden, Menorrhagien, Hämorrhagien, Leukorrhoe und Ulzerationen angewendet [29].

Inhaltsstoffe sind monomere und dimere Ellagitannine, Flavonoide, Phenolsäuren, Steroide, Cumarine und Spuren von Alkaloiden [29], [30]. In neueren pharmakologischen Untersuchungen konnten für Lythri herba bzw. daraus hergestellte Extrakte in vitro und tierexperimentell antidiarrhoische, antioxidative, entzündungshemmende und antimikrobielle Wirkungen nachgewiesen werden, die auf den hohen Gehalt an Gerbstoffen und anderen phenolischen Verbindungen zurückführbar sein dürften und die überlieferten Anwendungsgebiete plausibel machen. Nennenswerte unerwünschte Wirkungen bei der Anwendung sind nicht bekannt [30]. Heute findet der Blutweiderich noch Anwendung im Nahen Osten

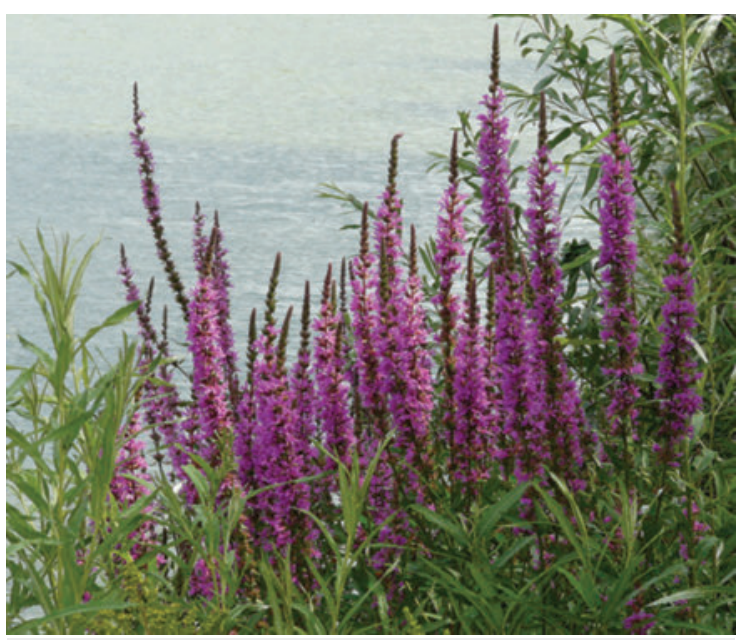

Abb. 1 Blutweiderich (Lythrum salicaria) am Rheinufer. Quelle: Manfred Heyde
(Iran, Jordanien), Russland und einigen europäischen Ländern (Italien, Rumänien, Bosnien-Herzegowina), insbesondere innerlich bei gastrointestinalen Beschwerden, selten auch bei Hauterkrankungen (z. B. in Italien) [29].

Der Gewöhnliche Blutweiderich wurde in Frankreich von der ländlichen Bevölkerung seit langem geschätzt [30]. So merkte auch Madaus 1938 an, dass in Frankreich dem Blutweiderich mehr Beachtung geschenkt werde [26]. Im 18. bis zum 20. Jahrhundert wurden insbesondere in Frankreich Berichte über die Wirkungen von L. salicaria veröffentlicht, u.a. als Fallberichte zur Wirkung bei Dysenterien, aber auch zu einer Zubereitung namens „Salicairine“, 1920 von Dumont zur Behandlung von Diarrhö bei Kindern, unspezifischer Enteritis, bakterieller Enteritis, Dysenterie sowie in Kombination mit Emetin bei Amöbiasis eingesetzt [26], [29]. In Frankreich ist derzeit weiterhin das Produkt „Salicairine“ als Lösung zum Einnehmen als traditionell registriertes Fertigarzneimittel für die (kurzzeitige) symptomatische Behandlung von Diarrhö erhältlich; in $100 \mathrm{~g}$ Produkt sind 66,66 g eines hydroalkoholischen Fluidextrakts der oberirdischen Teile von L. salicaria enthalten [2]. Ein weiteres Produkt, Élusanes salicaire, mit $200 \mathrm{mg}$ eines Trockenextraktes aus Lythri herba pro Kapsel (Trockenextrakt mit DEV 4-6:1, Extraktionsmittel Ethanol-Wasser 50\% (V/V)) ist in Frankreich für die gleiche Indikation als traditionelles Arzneimittel registriert [2]. Volksmedizinisch wird Blutweiderich in Frankreich auch peroral bei venöser Insuffizienz, Hämorrhoiden, zur symptomatischen Behandlung von Diarrhö sowie zur analgetischen Behandlung im Mundund Rachenraum in Form eines Tees eingesetzt [13]. Ausgehend von einer Monografie im französischen Arzneibuch ist seit 2013 Blutweiderichkraut, Lythri herba, mit einer Monografie im Europäischen Arzneibuch vertreten. Als Qualitätskriterium wird ein Mindestgehalt von 5,0\% Gerbstoffen, berechnet als Pyrogallol und bezogen auf die getrocknete Droge, gefordert [12]. In Frankreich gehört Lythri herba zu den Drogen, die derzeit für eine traditionelle Anwendung zugelassen sind [3]. Monografien des HMPC, der ESCOP und/oder der Kommission E liegen nicht vor.

\section{Die Blutwurz-Potentilla erecta L.}

Die aus $P$. erecta gewonnene Rhizomdroge, Tormentillae rhizoma, oftmals auch als Tormentillae radix bezeichnet, war bereits in der Antike bekannt. In Kräuterbüchern des Mittelalters, u.a. von Leonhart Fuchs und Hildegard von Bingen, wird die Anwendung der Rhizomdroge als Blutstillmittel, als Heilmittel bei Entzündungen, besonders im Mund- und Rachenraum, bei Fieber, Durchfall, Ruhr und Pest erwähnt [25]. P. erecta aus der Familie der Rosaceae ist in fast allen Teilen Europas beheimatet. Die mehrjährige Staude fällt durch die 4-zähligen, gelben Blüten und durch die gefingerten, dunkelgrünen Blätter auf ( $\triangleright$ Abb. 2). Charakteristisch ist der Wurzelstock, der 


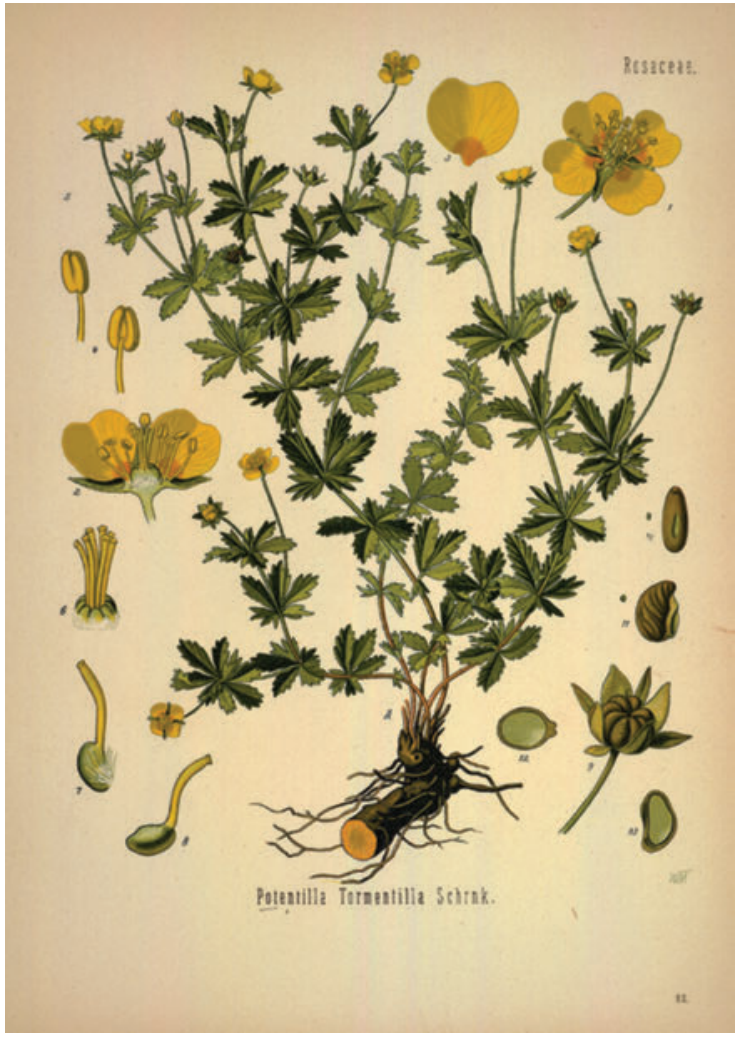

-Abb.2 Potentilla erecta (Blutwurz) aus Köhlers Medizinalpflanzen [20].

fingerdick, knollig und walzenförmig ist. Inhaltsstoffe in der Rhizomdroge sind kondensierte und hydrolysierbare Gerbstoffe, Flavonoide und weitere phenolische Verbindungen sowie Triterpene [25]. Neue In-vitro- und tierexperimentelle Untersuchungen führten zum Nachweis von anti-inflammatorischen, antibakteriellen, antiviralen und antidiarrhoischen Wirkungen, die damit die Indikationen der Antike und des Mittelalters plausibel erscheinen lassen [25], [34]. Hervorzuheben ist, dass in einer klinischen Studie bei Kindern ein Extrakt aus P. erecta Durchfälle, die durch Rotaviren verursacht waren, im Hinblick auf die Dauer der Durchfälle und auf das Ausmaß der damit einhergehenden Dehydrierung verringern konnte [33]. Ebenso ist eine klinische Studie zur Anwendung bei Colitis ulcerosa bemerkenswert: Ein Extrakt führte bei den meisten der 16 Studienteilnehmern zu einer kompletten Remission, die Steroid-Begleitmedikation konnte reduziert oder sogar weggelassen werden [19]. In einer weiteren klinischen Studie zeigte sich, dass ein Extrakt zur Verringerung und Beseitigung der Symptome bei chronischer Gastroduodenitis beitragen kann und eine Reduktion der Entzündung des Magens und des Duodenums bewirkt [16].

Bis zum Jahr 2010 war mit Blutwurz-ratiopharm ein zugelassenes Fertigarzneimittel in Deutschland verkehrsfähig, das bei unspezifischen Durchfallerkrankungen und nur für eine kurzzeitige Anwendung bestimmt war; es enthielt $200 \mathrm{mg}$ Tormentillwurzelextrakt, gewonnen mit einem DEV von 3,5-4,5:1 und dem Extraktionsmittel Ethanol $60 \%$ (V/V) [7]. Derzeit liegt in Deutschland eine Standardzulassung für einen Tee, der aus Tormentillwurzelstock bereitet wird, vor; Indikationen sind die symptomatische Behandlung von leichtem Durchfall und die symptomatische Behandlung von leichten Entzündungen der Mundschleimhaut (bei lokaler Anwendung). Aktuell ist in Deutschland ein Kombinationspräparat als zugelassenes Fertigarzneimittel im Handel, das u.a. auch einen P.-erecta-Extrakt enthält und bei Mundfäule (Stomatitis aphthosa) eingesetzt wird.

Tormentillwurzelstock ist im Europäischen Arzneibuch mit einer Monografie vertreten, sodass die Anforderungen an die Qualität der Arzneidroge festgelegt sind: Ein Mindestgehalt von 7\% Gerbstoffen, berechnet als Pyrogallol und bezogen auf die getrocknete Droge, wird gefordert; in einer weiteren Monografie werden die Qualitätsanforderungen an eine Tormentilltinktur festgelegt [12]. Eine Monografie der Kommission E zur Anwendung der Tormentillwurzeln liegt vor [22]. In einer Monografie des HMPC der EMA wurde aufgrund der vorliegenden Daten der „traditional use“ für Zubereitungen aus Tormentillae rhizoma befürwortet; berücksichtigt wurden die zerkleinerte Droge, Tinkturen und/oder Flüssig- und Trockenextrakte, die zur symptomatischen Behandlung leichter Diarrhö oder bei der symptomatischen Behandlung kleinerer Entzündungen im Bereich der Mundschleimhaut eingesetzt werden [18]. Die ESCOP veröffentlichte ebenfalls für diese Indikationen eine Monografie und äußerte ebenso, dass die Wirksamkeit aufgrund der Erfahrung und langjährigen Anwendung plausibel sei [11].

\section{Echter Alant-Inula helenium L.}

Der Echte Alant ist auch unter verschiedenen anderen deutschen Bezeichnungen bekannt, u.a. Brustalant, Darmwurz oder Helenenkraut und Großer Heinrich. Bei dieser Asteraceae handelt es sich um eine ausdauernde, $80-180 \mathrm{~cm}$ hohe Pflanze mit einem kräftigen Wurzelstock und langen Wurzeln (bis $50 \mathrm{~cm}$ lang). Die Stängel sind aufrecht, die Blätter haben eine Größe von 15$25 \mathrm{~cm} \times 40-80 \mathrm{~cm}$. Die Blütenköpfchen sind $6-7 \mathrm{~cm}$ breit, zahlreich und krautig angeordnet. Auffallend sind die kräftig gelben Blüten mit Zungen- und Röhrenblüten ( $\triangleright$ Abb.3), die zwischen Juni und Oktober erscheinen [17].

Ursprünglich stammt $I$. helenium offenbar aus Zentralasien, ist heute aber in Mitteleuropa auch heimisch und wächst an Wiesengräben und an Weg- und Waldrändern. Als Droge wird bzw. wurde die Rhizomdroge („Alantwurzel“) arzneilich verwendet, die sog. Helenii Radix (auch Radix Enulae und Radix Inulae). Ausschließlich im Ergänzungsband zum DAB 6 (Ausgabedatum 1941) waren die 


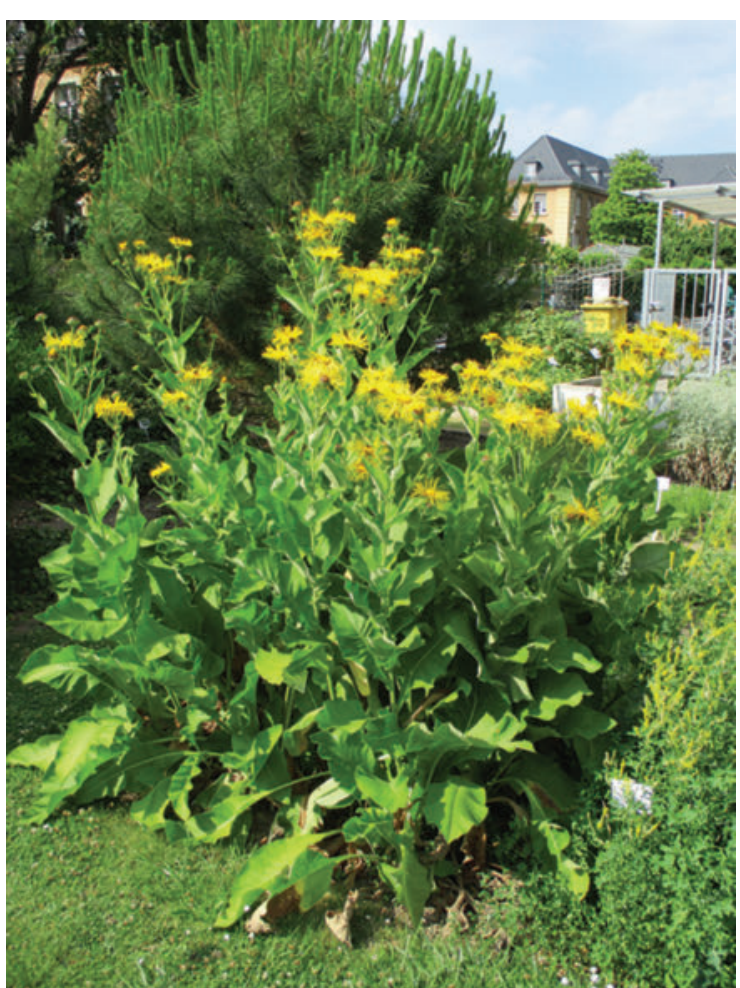

-Abb.3 Alant (Inula helenium) im Botanischen Garten Bonn (Nutzpflanzengarten). Quelle: K.P. Latté
Alantwurzel und die Zubereitung Alantwurzelextrakt monografiert: Bei letzterer handelt es sich um einen alkoholisch-wässrigen, viskosen Extrakt [9].

Inhaltsstoffe in Alantwurzel sind ein ätherisches Öl, wobei hierin Sesquiterpenlactone vom EudesmanolidTyp dominieren: Alantolacton und Isoalantolacton sowie deren Dihydroverbindungen und Tetrahydroalantolacton sind neben weiteren Eudesmanoliden in kleineren Mengen enthalten. Alantolacton und seine Derivate zeichnen sich strukturell durch eine exozyklische Doppelbindung aus, die offenbar mit dem Auftreten von allergischen Reaktionen in Verbindung steht (s.u.). Weitere Stoffe in Alantwurzel sind Terpene, Harze, Wachse und Polysaccharide (Inulin!) [17].

In der Antike war der Echte Alant als Heilmittel und als Lebensmittel bekannt [20]. Im Kräuterbuch von L. Fuchs werden für Alantwurzel folgende Wirkung beschrieben: harntreibende und emmenagoge Wirkungen, außerdem wird Alantwurzel bei Husten und Blutspeien als Latwerge (Extrakt-Honig-Zubereitung) angewendet, ferner bei Magenbeschwerden. Die Blätter des Echten Alant wurden äußerlich angewendet, u. a. bei Geschwüren [15]. Ende des 19. Jahrhunderts war, beschrieben durch Köhler, neben der Gewinnung von Inulin aus 
Alantwurzel die Anwendung bei folgenden Indikationen bekannt: in Latwergen und als Extrakt innerlich bei Hustenreiz, äußerlich in Abkochungen oder als Zusatz zu Salben bei Hautausschlägen und Krätze [20]. Köhler weist 1887 bereits darauf hin, dass Echter Alant nur noch wenig verwendet werde. Neben der Droge wurde ein Extrakt (Extractum Helenii) sowie als Zubereitungen Tinctura Helenii, Ptisana Helenii und Vinum Helenii arzneilich eingesetzt. In einzelnen Mitteilungen des 19. Jahrhunderts wurde über die Anwendung von Alantwurzel bei Tuberkulose berichtet [26]. Verschiedene Rezepturen waren in den 1930er-Jahren bekannt, u.a. ein wässriger Extrakt bei „Verschleimung der Respirations- und Verdauungsorgane" sowie eine Alantsalbe bei Krätze (Wurzeldroge zu Brei gekocht, mit Butter vermischt) [26].

Moderne pharmakologische Untersuchungen belegen in vitro antimikrobielle Wirkungen, die wohl besonders auf dem Gehalt an Sesquiterpenen beruhen [17]. Tierexperimentell konnten anthelminthische Wirkungen gezeigt werden, die ebenfalls auf diese Naturstoffe zurückzuführen sind. Für einzelne Sesquiterpenlactone konnten in vitro und tierexperimentell antitumorale Wirkungen nachgewiesen werden [17].

Die Kommission E kam 1988 zu dem Ergebnis, dass die Wirksamkeit der Droge und ihrer Zubereitungen bei den beanspruchten Anwendungsgebieten (innerliche Anwendung bei Erkrankungen und Beschwerden im Bereich der Atemwege, des Magen-Darm-Traktes sowie der Niere und der ableitenden Harnwege) nicht ausreichend belegt sei; angesichts des Risikos einer Allergie könne die therapeutische Anwendung nicht befürwortet werden. Hierzu führte die Kommission E aus, dass die Sequiterpenlactone die Schleimhäute reizen würden, sensibilisierend wirken und allergische Kontaktdermatitiden hervorrufen würden. Alantolacton werde als Hapten an Hautproteine gebunden, und das Addukt induziere eine Überempfindlichkeitsreaktion gegenüber Alantolacton und weiteren strukturverwandten Verbindungen. Größere Gaben würden zu Erbrechen, Durchfällen, Krämpfen und Lähmungserscheinungen führen [23]. Offenbar beeinflusst bei den sensibilisierenden bzw. reizenden Effekten der Alantwurzel die Art der Zubereitung das Ausmaß, das bei einem Tee wohl am geringsten ist, gefolgt von einem Trockenextrakt (Wasser); am stärksten waren diese Effekte bei einer Tinktur und einem flüssigen Extrakt, hergestellt mit 70\%igem Ethanol [32].

Monografien des HMPC und der ESCOP liegen nicht vor. In Frankreich gehört die Alantwurzel zu den Drogen, die für eine traditionelle Anwendung zugelassen sind [3]. Im Französischen Arzneibuch ist Alantwurzel, bestehend aus den Wurzeln und Rhizomen von I. helenium, heute nach wie vor unter der Bezeichnung „Aunée“/,Inula helenium“ monografiert [28]. Ebenso ist die Alantwurzel im
Russischen Arzneibuch als „Rhizomata et Radices Inulae“ mit einer Monografie enthalten. Alantwurzel wird in Russland nach wie vor bei Erkältungskrankheiten angewendet, u.a. als standardisierter, klinisch geprüfter Extrakt in dem zugelassenen Arzneimittel „Alanton“ [32].

\section{Leberblümchen - Hepatica triloba L.}

Leberblümchen aus der Familie der Ranunculaceae sind ausdauernd und werden $5-15 \mathrm{~cm}$ hoch und bilden unterirdisch einen Wurzelstock. Charakteristisch sind die ledrigen, herzförmigen, dreilappig geteilten Blätter, die erst nach der Blüte erscheinen und oben grün, unterseits violett sind [17], [26]. Auffallend sind im März und April die 6-8 himmelblauen Blütenhüllblätter ( A Abb.4). H. nobilis ist in fast ganz Europa heimisch und steht in Deutschland, Österreich und der Schweiz wie auch in anderen europäischen Ländern unter Artenschutz. Arzneilich verwendet wurde die Krautdroge, Hepaticae herba (oder auch Herba et Flores Hepaticae). Inhaltsstoffe der Krautdroge sind lactonbildende Glucoside, wobei Ranunculin (Glykosid) sehr leicht in Protoanemonin gespalten wird, das seinerseits unter Bildung von Anemonin dimerisiert. Protoanemonin ist in der getrockneten Droge nicht mehr enthalten [17]. Weitere Inhaltsstoffe der Krautdroge sind Flavonolglykoside, Anthocyane, ein Saponin und ein Alkaloid [17].

In der Antike und auch noch zu Zeiten von Paracelsus (1493-1541) wurden in der sogenannten Signaturenlehre Pflanzen, die einem erkrankten Körperorgan des Menschen ähnlich sahen, als geeignetes Mittel angesehen, im Falle des Leberblümchens die Anwendung bei Leberbeschwerden. In den Kräuterbüchern des 16. Jahrhunderts von $\mathrm{H}$. Bock, Matthiolus und Tabernaemontanus werden die heilende Wirkung bei Leber-, Nieren- und Blasenbeschwerden und bei Wunden als Anwendungsgebiete von Leberblümchenkraut genannt; u.a. wurde dazu eine Zubereitung aus dem Kraut in Wein verwendet (,in Wein gesotten“) [26]. Noch im 18. Jahrhundert wurde Hepaticae herba als Lebertherapeutikum verwendet, und zwar als Trank, Tee oder Kräuterwein. Seit dem 19. Jahrhundert

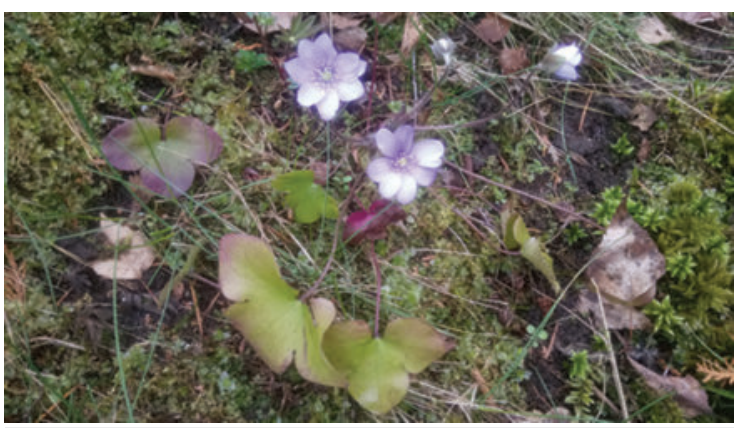

Abb. 4 Leberblümchen (Hepatica nobilis), Berlin. Quelle: K.P. Latté 


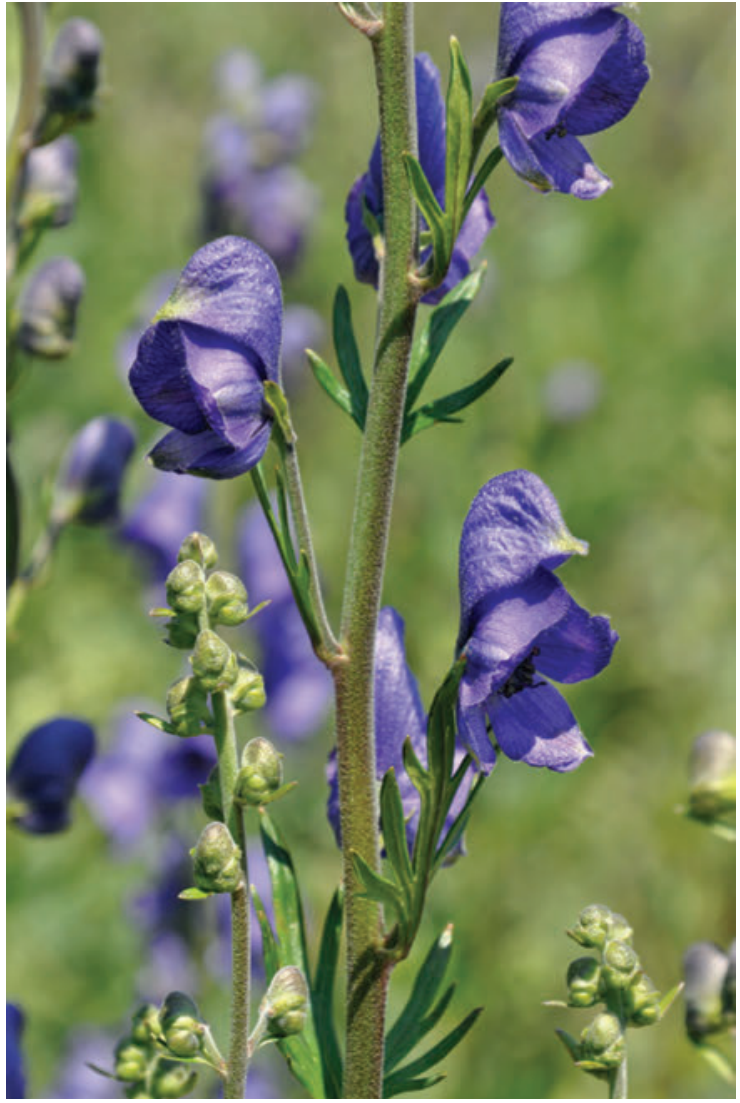

-Abb. 5 Blühender Eisenhut. Quelle: naturganznah

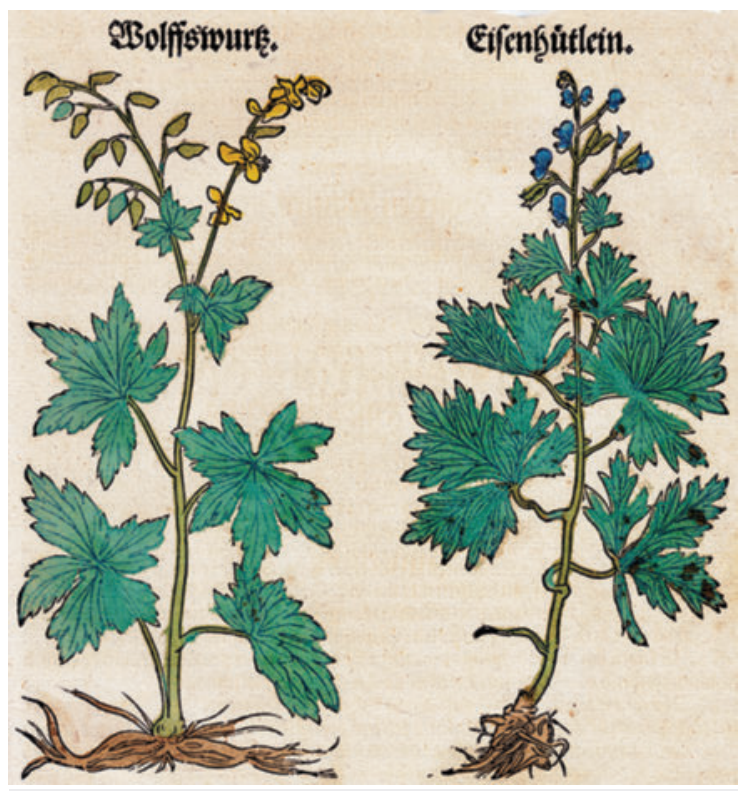

-Abb. 6 Aconitum vulparia („Wolffswurtz“) und A. napellus („Eisenhütlein“). Abbildung aus dem Kräuterbuch von Hieronymus Bock von 1546.

hat diese Heilpflanze an Bedeutung verloren und wurde allenfalls volksmedizinisch angewendet [1], u.a. innerlich bei Hepatopathien und bei Nieren- und Blasenleiden sowie äußerlich bei Wunden, Geschwüren, rheumatischen Erkrankungen, Mandelentzündung, bei Schmerzen und als „blasenziehendes“ Mittel. Dabei erfolgte die innerliche Anwendung als Infus, Mazerat in Wasser oder Wein, während bei äußerlicher Anwendung die zerquetschte, frische Pflanze verwendet wurde [17]. Der Kontakt von Protoanemonin mit der Haut und Schleimhaut, insbesondere bei Anwendung der frischen Pflanze, führt zu heftigen lokalen Reaktionen, die bei der getrockneten Droge jedoch nicht zu erwarten sind [17]. Die Kommission E erstellte für Leberblümchenkraut eine Negativmonografie und verwies auf die nicht belegte Wirksamkeit und die Risiken von Protoanemonin-haltigen Zubereitungen. Bei innerlicher Anwendung könnten bei höherer Dosierung Reizungen der Nieren und der ableitenden Harnwege auftreten [24]. Heute ist Hepaticae herba als Phytotherapeutikum obsolet und findet nur noch in der Homöopathie Anwendung. Klinische Studien oder andere Belege zur Wirksamkeit liegen nicht vor.

\section{Blauer Eisenhut-Aconitum napellus L.}

Die Gattung Aconitum gilt als giftigste Pflanzengattung Europas [6]. Eine dieser Aconitum-Arten, A. napellus, der Blaue Eisenhut oder Sturmhut, kommt in Gebüschen, auf Schutthalden und auf feuchten, humosen Weiden und an Bachläufen vor. Der Blaue Eisenhut wird bis zu $1,5 \mathrm{~m}$ hoch, hat ein schwarzes, fleischiges und rübenartiges Rhizom. Charakteristisch sind die 3- bis 7-teilig handförmigen Blätter und die 5 violetten Kronblätter, wobei der sogenannte „Helm“, das oberste Blütenblatt, wie ein Sturmhut 2 langgestreckte Honigblätter umschließt ( Abb.5, 6) [20], [26]. Aconitum war bereits in der Antike bekannt. Im 16. Jahrhundert wurde Aconitum als tödliches Gift betrachtet. So berichtet Matthiolus (1501-1577) von der Giftigkeit, während Lonicerus (1528-1586) und Bock in ihren Kräuterbüchern die Samen aufgrund einer speicheltreibenden und emetischen Wirkung sowie die Wurzel und das Kraut nur äußerlich bei faulem Zahnfleisch und in einer Läusesalbe anwenden. Zu Beginn des 18. Jahrhunderts wurde Aconitum bei Lepra, Wechselfieber und Augenschmerzen eingesetzt, erst etwas später folgte auch die Anwendung bei neuralgischen und rheumatischen Schmerzen [26]. Im 19. bis hinein in das 20. Jahrhundert erfolgte die Anwendung bei Rheuma, Gicht, Migräne und Trigeminusneuralgien [20], [26]. Arzneilich fanden die Wurzelknolle, Tubera Aconiti, und die Blätter, Folia Aconiti, Verwendung. Daraus wurden verschiedene Zubereitungen (u.a. Aconitinum, ein Extrakt, ein Liniment und ein Sirup) hergestellt. Allerdings wurde immer vor tödlichen Vergiftungen bei innerlicher Anwendung aufgrund der Verschiedenheit der Präparate gewarnt [20].

A. napellus war bis zur 5. Ausgabe des DAB, erschienen 1910 und gültig bis 1926, mit 2 Monografien 
>Tab. 1 „Wege“ von früher verwendeten Heilpflanzen bis in die heutige Zeit (ohne Anspruch auf Vollständigkeit; Fettdruck: die in diesem Beitrag besprochenen Pflanzen).

\section{Weg der Pflanzen/ Drogen bis heute}

Verdrängung durch Heilpflanzen aus anderen Kulturkreisen ab dem 18. Jahrhundert

Verdrängung durch synthetische Wirkstoffe

Nachweis von Inhaltsstoffen, die die Anwendung beeinträchtigen, daher keine oder kaum noch Verwendung

Pflanzen mit stark toxischen Inhaltsstoffen bzw. geringer therapeutischer Breite, daher keine (oder kaum noch) Verwendung

Schriften keine Bedeutung zugemessen bzw. „einfach“ vergessen

Heilpflanzen selbst nicht bzw. kaum mehr verwendet, aber daraus isolierte Einzelstoffe und davon abgeleitete Stoffe

Heilpflanze ausgestorben bzw. vom Aussterben bedroht (Artenschutz)

Anwendung „nur“ noch lokal begrenzt in der Volksmedizin

Wirksamkeit bei überlieferten Indikationen nicht belegt (und ggf. auch nicht plausibel)

Pflanzen mit Status „traditional use“, regional in einem EU-Land oder EU-weit, Registrierung als traditionelles Arzneimittel

Pflanzen mit Status „well-established use”, EU-weit, Zulassung als Arzneimittel

Anwendung „nur“ noch in der Homöopathie

Verwendung „nur“ noch als Lebensmittel bzw. Gewürz bekannt

Verwendung bzw. „Wiederentdeckung“ als sog. „Botanicals“ in Lebensmitteln, u. a. in Nahrungsergänzungsmitteln*

* Überprüfung der Zulässigkeit ist Aufgabe der zuständigen Behörden
Beispiele für Pflanzen (bzw. deren Drogen)

Echter Eisenhut (Aconitum napellus) und Weißer Senf (Sinapis alba) z. B. durch Teufelskralle (Harpagophytum sp.) bei der Rheumabehandlung

diuretisch wirkende Pflanzen wie die Wilde Möhre (Daucus carota), die Sandsegge (Carex arenaria) oder der Besenginster (Cytisus scoparia) durch synthetische Diuretika

Echter Alant (Inula helenium)

Echter Eisenhut (Aconitum napellus), Roter Fingerhut (Digitalis purpurea)

Kleine Braunelle (Prunella vulgaris), Kriechendes Fingerkraut (Potentilla reptans)

Weidenrinde (Salix sp.): Entwicklung von Acetylsalicylsäure; Tollkirsche (Atropa belladonna): Atropin

ausgestorben: „Silphon“ aus der Antike; unter Artenschutz: Sonnentau (Drosera sp.), Edelweiß (Leontopodium alpinum)

Edelweiß (Leontopodium alpinum) im Alpenraum

Leberblümchen (Hepatica nobilis)

Blutweiderich (Lythrum salicaria) in Frankreich; Blutwurz (Potentilla erecta) EU-weit

Baldrian (Valeriana officinalis), Sonnenhut (Echinacea purpurea)

\section{Echter Eisenhut (Aconitum napellus)}

Gewürz: Oregano (syn. Dost, Origanum vulgare); Lebensmittel: Quitte (Cydonia oblonga)

Weißdornblätter-Extrakt (Crataegus sp.); Mischung aus Baldrianwurzel-Pulver (Valeriana officinalis) und Melissenblätter-Pulver (Melissa officinalis) vertreten: mit den Tubera Aconiti (Eisenhutknollen) und der Tinctura Aconiti (Eisenhuttinktur), bereitet aus 1 Teil gepulverter Droge und 10 Teilen verdünntem Weingeist [8].

Inhaltsstoffe in allen Teilen des Eisenhuts sind Diterpenalkaloide, die für die pharmakologischen und toxikologischen Wirkungen verantwortlich sind: so sind NorDiterpenalkaloide (z. B. Aconitin, Benzoylaconitin, N-Desethylaconitin, Meaconitin) sowie echte Diterpenalkaloide enthalten [6]. Erste Anzeichen einer Vergiftung nach oraler Aufnahme geringer Mengen treten nach wenigen Minuten auf und erreichen je nach Zufuhrmenge unterschiedliche Ausprägungen, im Extremfall auch den Tod [6]. Die letale Dosis beträgt 3-6 mg Aconitin, die bereits mit wenigen Gramm des Pflanzenmaterials erreicht werden [14]. Aconitin bindet an spannungsabhängige Natriumkanäle von erregbaren Membranen, was deren
Inaktivierung hemmt. An Nervenzellen führt das zu Lähmungen [6]. Vergiftungsfälle von Kindern mit AconitumPflanzen treten heute immer wieder auf, im Zeitraum von 1998 bis 2004 gab es in Deutschland 86 Vergiftungsfälle [6].

Die Kommission E erstellte 1987 eine Negativmonografie zu Aconiti tuber und Aconiti herba. Beanspruchte Indikationen waren Schmerzen, Fazialislähmung, Gelenkerkrankungen, Rheuma, Gicht, rheumatische Beschwerden, Pleuritis, Pericarditis sicca, Fieber, Haut- und Schleimhauterkrankungen sowie die Anwendung zur Desinfektion und Wundbehandlung. Die Kommission E verwies auf die geringe therapeutische Breite, sodass Intoxikationserscheinungen bereits im therapeutischen Dosisbereich auftreten könnten, u. a. Parästhesien, Erbrechen, Schwindel, Muskelkrämpfe, Hypothermie, Bradykardie und Herzrhythmusstörungen und zentrale Atemlähmung. Daher 
sei die Anwendung aufgrund dieser Risiken nicht zu vertreten [21]. Heute ist die Anwendung von AconitumZubereitungen, abgesehen von homöopathischen Zubereitungen, obsolet.

\section{Fazit}

Aus heutiger Sicht haben die vielen Pflanzen und pflanzlichen Drogen, die in der Antike und im Mittelalter bekannt und als Heilmittel eingesetzt wurden, ganz unterschiedliche Wege genommen ( $\triangleright$ Tab. 1). Einige der lange bekannten Heilpflanzen werden heute in der Phytotherapie im Rahmen einer „traditionellen Anwendung“ (sog. „traditional use“) verwendet, u.a. als Teezubereitungen oder auch als Extrakte, wobei die Wirksamkeit aufgrund der Erfahrung und der langjährigen Anwendung als erwiesen angesehen wird. Jedoch wäre es sehr erstrebenswert, wenn klinische Studien zu diesen Drogen durchgeführt würden, um die Wirksamkeit im Sinne der rationalen Phytotherapie zu belegen und den Status des sog. „well-established use“ zu rechtfertigen. Die moderne Phytotherapie grenzt sich damit bewusst ab von der jahrtausendealten Heilpflanzenkunde, in der teilweise die Humorallehre oder die Signaturenlehre, aber auch Magie und Aberglaube, Grundlage für die Zuordnung von bestimmten Wirkungen zu Arzneipflanzen waren und das Wissen durch Überlieferung von Generation zu Generation weitergegeben wurde.

Die traditionell verwendeten Drogen Blutweiderichkraut und Tormentillwurzelstock bzw. deren Extrakte weisen ein ähnliches phytochemisches Inhaltsstoffspektrum auf und zeigen, dass gerade bei der Therapie von leichten Fällen von Dysenterien und Durchfall eine im Vergleich zu synthetischen Wirkstoffen gute Alternative mit weniger unerwünschten Wirkungen zur Verfügung steht. Ausgehend von einer ersten Studie zu einem Tormentillwurzelstockextrakt könnte dies möglicherweise auch für die Behandlung von Colitis ulcerosa gelten, da die Therapie bei dieser Indikation mit den synthetischen Wirkstoffen wie Kortikosteroiden und Sulfasalazin mit erheblichen Nebenwirkungen verbunden ist.

Im Falle von Alantwurzel stehen sich die Bewertung der deutschen Kommission E, die auf das Auftreten von allergischen Reaktionen in einer Negativmonografie hingewiesen hat, und die derzeitige traditionelle Verwendung als Arzneimittel in Frankreich sowie die Anwendung in Russland gegenüber. Da möglicherweise die sensibilisierenden Effekte abhängig von der Art der Zubereitung sind, könnte eine erneute Beurteilung des Nutzen-RisikoVerhältnisses infrage kommen. 
In jedem Fall müssen die Risiken, die mit der Anwendung der einzelnen pflanzlichen Drogen verbunden sind, berücksichtigt werden. Das Zitat von Paracelsus (1493-1541): „Alle Wiesen und Matten, alle Berge und Hügel sind Apotheken. Die natürliche Apotheke übertrifft die menschliche" sollte daher keinesfalls wortwörtlich genommen werden. Jede Pflanze ist einzeln zu betrachten, da das alte Wissen nicht unkritisch übernommen werden kann. Die volksmedizinische Anwendung von Pflanzen mit teils unverantwortlichen Indikationen durch nicht ausreichend ausgebildete Personen (sog. „Heilkräuterspezialisten“) ist als problematisch anzusehen, sodass insbesondere Ärzten und Apothekern bei der Anwendung von „vergessenen“ Heilpflanzen eine besondere Verantwortung im Hinblick auf die Beratung der Patienten zukommt [31], um diese vor etwaigen schädlichen Wirkungen zu schützen.

Aus Sicht der Phytotherapie sind neuere Tendenzen kritisch zu sehen, dass altbekannte Heilpflanzen als sog. „Botanicals“ „wiederentdeckt“ und als pflanzliche Zutat in Lebensmitteln, wie z.B. in Nahrungsergänzungsmitteln, verwendet werden und unzulässigerweise bei gesundheitsbezogenen Angaben und beim Nachweis einer langjährigen Verwendung als Lebensmittel ein Bezug zur traditionellen Anwendung als Heilpflanze gezogen wird. Jeder Fall ist von den zuständigen Behörden einzeln zu betrachten.

Insgesamt handelt es sich bei den „vergessenen“ Heilpflanzen um einen Schatz, der unbedingt (weiter) gehoben werden sollte, wie dies bereits u.a. im Rahmen des Projektes „Klostermedizin“ [27] erfolgt. Da pflanzliche Drogen und ihre Extrakte als Vielstoffgemische an verschiedenen „targets“ ansetzen und zu pleiotropen Wirkungen im Körper führen, könnten diese eine besser verträgliche und nebenwirkungsärmere Alternative zu synthetischen Wirkstoffen bei ausgewählten Indikationen sein und die heutige Phytotherapie sinnvoll erweitern.

\section{ABSTRACT}

\section{Forgotten medicinal plants}

Plants have been used at all times of mankind as food and as remedies for ailments. In antiquity and later in medieval times, a lot of plants and their medicinal use were described. Due to various reasons, including the advent of synthetic drugs starting from the $19^{\text {th }}$ century, knowledge of medicinal plants has been partially lost. In this article, the path of five herbal remedies, namely the herbal parts of purple loosestrife, the tormentil rhizomes, elecampane root, liverwort leaves and the tubers of monkshood, are followed from antiquity to the present day and their current uses are illuminated.

\section{Keywords}

Lythrum salicaria, Potentilla erecta, Inula helenium, Hepatica nobilis, Aconitum napellus, forgotten medicinal plants, traditional use, well-established use, rational phytotherapy.

Interessenkonflikt

Der Autor erklärt, dass kein Interessenkonflikt besteht.

\section{Korrespondenzadresse}

\author{
Dr. Klaus Peter Latté \\ Warägerweg 21 \\ 13595 Berlin \\ Deutschland \\ E-Mail: latte@zedat.fu-berlin.de
}

\section{Literatur}

[1] Anagnostou S. Pflanzliche Lebertherapeutika. Von Leberblümchen und Geelwurtz. Pharmazeutische Zeitung 2011; 156: 58-65

[2] ANSM. Résumé des caractéristiques du produit. Dokument der Agence nationale de sécurité du médicament et des produits de santé; Eintrag zu Produkt "Salicairine“ vom 10.12.2013, Link: http://agence-prd.ansm.sante.fr/php/ecodex/rcp/R0233 743.htm, und Eintrag zu Produkt „Élusanes salicaire“ vom 19.08.2010, Link: http://agence-prd.ansm.sante.fr/php/eco dex/rcp/R0177630.htm, eingesehen am 09. 03.2020

[3] ANSM. Liste A des plantes médicinales utilisées traditionnellement. Dokument der Agence nationale de sécurité du médicament et des produits de santé; Pharmacopée française, Januar 2020, Link: https://www.ansm.sante.fr/var/ansm_site/ storage/original/application/dc6398f1f676936f296909ec52f c2213.pdf, eingesehen am 09.03.2020

[4] Bauer Petrovska B. Historical review of medicinal plants' usage. Pharmacogn Rev 2012; 6: 1-5

[5] Beiser R. Vergessene Heilpflanzen. Botanik, Volksheilkunde, Anwendungen. 2. Aufl. Aarau und München: AT-Verlag; 2017

[6] BfR-Bundesinstitut für Risikobewertung. Risikobewertung von Pflanzen und pflanzlichen Zubereitungen. Klenow S, Latté KP, Wegewitz U, Dusemund B, Pöting A, Appel KE, Großklaus R, Schumann R, Lampen A, Hrsg. BfR Wissenschaft 01/2012, Kap. 10: Aconitum spp. (Eisenhut-Arten). Seiten 205-211

[7] Blutwurz-ratiopharm. Gebrauchsinformation, Stand Februar 2005

[8] DAB 5. Monographien „Tinctura Aconiti. - Eisenhuttinktur.“ (S.517) und „Tubera Aconiti. - Eisenhutknollen.“ (Seite 547). Berlin: R. v. Deckers Verlag; 1910

[9] DAB 6 Ergänzungsbuch, 1941; als Neudruck („Ergänzungsbuch zum Deutschen Arzneibuch"). Berlin und Frankfurt a.M.: Deutscher Apotheker-Verlag; 1948

[10] Dressendörfer W. Blüten, Kräuter und Essenzen. Heilkunst alter Kräuterbücher. Lizenzausgabe für die Wissenschaftliche Buchgesellschaft. Ostfildern: Thorbecke Verlag; 2003

[11] ESCOP. Monograph „Tormentillae rhizoma, Tormentil“. European Scientific Cooperative on Phytotherapy, Online Series, 2013 
[12] Europäisches Arzneibuch. 9. Ausgabe: Monographien 1537/ „Blutweiderichkraut, Lythri herba“, 1478/ „Tormentillwurzelstock, Tormentillae rhizoma“ und 1895/ „Tormentilltinktur, Tormentillae tinctura“

[13] Fleurentin J, Weniger B. Un tour du monde des plantes qui soignent. Afrique, Amériques, Chine, Outremer, Europe. Rennes: Éditions Ouest-France, 2018

[14] Frohne D, Pfänder HJ. Giftpflanzen. 5. Aufl. Stuttgart: Wissenschaftliche Verlagsgesellschaft; 2004, Monographie Aconitum

[15] Fuchs L. New Kreüterbuch. Basel; 1543. Kapitel 89 („Von Alantwurtz")

[16] Glubochenko OV. [Clinical efficiency of applications of Potentilla erecta preparation in complex treatment of patients with chronic gastroduodenitis accompanied by noncalculous cholecystitis]. Acta Med Leopoliensia 2001; 1: 43-46. Abstract englisch

[17] Hagers Enzyklopädie der Arzneistoffe und Drogen. 6. Aufl. Stuttgart: Wissenschaftliche Verlagsgesellschaft; 2007, Monographien Inula und Hepatica

[18] HMPC. Community herbal monograph on Potentilla erecta (L.) Raeusch., rhizoma. Committee on Herbal Medicinal Products der EMA, Dokument EMA/HMPC/5513/2010 vom 25. 11.2010

[19] Huber R, Ditfurth AV, Amann F et al. Tormentil for active ulcerative colitis: an open-label, dose-escalating study. J Clin Gastroenterol 2007; 41: 834-838

[20] Köhler FE. Köhler's Medizinal-Pflanzen in naturgetreuen Abbildungen mit kurz erläuterndem Texte. Verlag GeraUntermhaus 1887, Band 1, 72, Monographie „Aconitum Napellus L." und Band 2, 90, Monographie "Inula Helenium L.“"

[21] Kommission E. Monographie „Aconitum napellus (Blauer Eisenhut)“ mit „Aconiti tuber, blaue Eisenhutknollen“ und Aconiti herba, blaues Eisenhutkraut“. Bundesanzeiger vom 15.10.1987, Nr. 193

[22] Kommission E. Monographie „Tormentillae rhizoma (Tormentillwurzelstock)“. Bundesanzeiger vom 5.5.1988, Nr. 85

[23] Kommission E. Monographie „Helenii radix (Alantwurzel)“. Bundesanzeiger vom 5.5.1988, Nr. 85

[24] Kommission E. Monographie „Hepatici nobilis herba (Leberblümchenkraut)“. Bundesanzeiger vom 14.7.1993, Nr. 128

[25] Latté KP. Potentilla erecta, Das Aufrechte Fingerkraut. Z Phytother 2006; 27: 198-206. doi: 10.1055/ s-2006-951594

[26] Madaus G. Lehrbuch der biologischen Heilmittel. Leipzig: Georg Thieme; 1938, Band 1: Monographie „Aconitum napellus. Eisenhut, Ranunculaceae“ (S. 388-400) und Band 2: Monographien „Hepatica triloba. Leberblümchen, Ranunculaceae“ (S. 1537-1541), „Inula helenium. Alant, Compositae“ (S. 1619-1626) und „Lythrum salicaria. Blutweiderich, Lythraceae“ (S. 1815-1818)

[27] Mayer JG. Klostermedizin-Heilwissen aus der Antike bis heute. Z Phytother 2019; 40: 254-258. doi: 10.1055 / a-10695248

[28] Pharmacopée française. Monographie „Aunée“, „Inula helenium“. Link: https://www.ansm.sante.fr/var/ansm_site/sto rage/original/application/734af3c3dabb05953e2d740776 c7e4ba.pdf, eingesehen am 09.03.2020

[29] Piwowarski JP, Granica S, Kiss AK. Lythrum salicaria L. - underestimated medicinal plant from European traditional medicine. A review. J Ethnopharmacol 2015; 170: 226-250. doi: 10.1016/j.jep.2015.05.017

[30] Piwowarski JP, Melzig MF. Der Gewöhnliche Blutweiderich, Lythrum salicaria L. Z Phytother 2016; 37: 275-279. doi: 10.1055 / s-0042-119179

[31] Schilcher H. Phytotherapie - Quo vadis? Pflanzenheilkunde zwischen Kräuterweibern, Ethno- und IQWIG-Botanikern. Gasteditorial. Z Phytother 2008; 29: 211-212. doi: 10.1055 / s-0028-1101524

[32] Shikov AN, Pozharitskaya ON, Makarov VG et al. Medicinal plants of the Russian Pharmacopoeia; their history and applications. J Ethnopharmacol 2014; 154: 481-536. doi: $10.1016 /$ j.jep.2014.04.007

[33] Subbotina MD, Timchenko VN, Vorobyov MM et al. Effect of oral administration of tormentil root extract (Potentilla tormentilla) on rotavirus diarrhea in children: a randomized, double blind, controlled trial. Pediatr Infect Dis J 2003; 22: $706-$ 710. doi: $10.1097 / 01$.inf.0000078355.29647.d0

[34] Tomczyk M, Latté KP. Potentilla -A review of its phytochemical and pharmacological profile. J Ethnopharmacol 2009; 122: 184-204. doi: 10.1016/j.jep.2008.12.022

Bibliografie

Zeitschrift für Phytotherapie 2020; 41: 1-7

DOI 10.1055/a-1126-9255

ISSN 0722-348X

(c) 2020. Thieme. All rights reserved.

Georg Thieme Verlag KG, Rüdigerstraße 14,

70469 Stuttgart, Germany 